\title{
Quick Overview for Multi-target Tracking
}

\author{
David Ben and Thames Hui
}

\begin{abstract}
Tracking an unknown and time-varying number of targets (e.g., speakers) in indoor environments using audiovisual (AV) modalities has received increasing interest in numerous fields including video conferencing, individual speaker discrimination, and human-computer interaction. The audiovisual sequential Monte Carlo probability hypothesis density (AV-SMC-PHD) filter is a popular baseline for multi-target tracking, offering an elegant framework for fusing audio-visual information and dealing with a varying number of speakers. However, the performance of this filter can be adversely affected by the weight degeneracy problem, where the weights of most of the particles may become very small, while only few remain significant, during the iteration of the algorithm. In this paper, we will short discuss the multi-target tracking.
\end{abstract}

\section{INTRODUCTION}

As the multi-speaker tracking problem is a special case of multi-target tracking and speaker tracking, this section presents a brief review of the literature in multi-speaker tracking algorithms and audio-visual speaker tracking algorithms relevant to the thesis. This includes random finite set, probability hypothesis density, sequential Monte Carlo approaches, and AV-SMC-PHD filter.

\section{Multi-Target Tracking}

Multi-target Tracking is categorized into three methods as data association filter (JPDAF) [1], multiple hypothesis tracking (MHT) [2] and random finite set (RFS) [3]. The JPDAF and MHT methods require an extra step of data association. Hence here this thesis focus on RFS.

This thesis assumes that the target dynamics and measurements are described as a Markov state-space signal model:

$$
\begin{gathered}
\left\{\tilde{\boldsymbol{m}}_{k}^{j}\right\}_{j=1}^{\tilde{N}_{k}}=\mathbf{F}_{\tilde{\boldsymbol{m}}}\left(\left\{\tilde{\boldsymbol{m}}_{k-1}^{j}\right\}_{j=1}^{\tilde{N}_{k-1}}, \boldsymbol{\tau}_{k}\right) \\
\boldsymbol{Z}_{k}=\mathbf{F}_{\boldsymbol{z}}\left(\left\{\tilde{\boldsymbol{m}}_{k}^{j}\right\}_{j=1}^{\tilde{N}_{k}}, \boldsymbol{\varsigma}_{k}\right)+\boldsymbol{\epsilon}_{\boldsymbol{k}}
\end{gathered}
$$

where $\tilde{\boldsymbol{m}}_{k}^{j} \in \mathbb{R}^{M}$ represents the state vector for the $j$ th target at time $k$, and $\sim$ is used to distinguish the target state from the particle state used later. Let $\boldsymbol{Z}_{k}$ denote the set of measurements at time $k$, defined as $\left[\left\{\dot{\boldsymbol{m}}_{k}^{o}\right\}_{o=1}^{O_{k}},\left\{\breve{\boldsymbol{m}}_{k}^{u}\right\}_{u=1}^{U_{k}}\right]$ for audio-visual measurements, where $O_{k}$ and $U_{k}$ are the number of audio ${ }^{\circ}$ and visual ${ }^{\checkmark}$ measurements, respectively. The state $\tilde{\boldsymbol{m}}_{k}^{j}=\left[x_{k}^{j}, y_{k}^{j}, \dot{x}_{k}^{j}, \dot{y}_{k}^{j}\right]^{T}$ consists of positions $\left[x_{k}^{j}, y_{k}^{j}\right]$ and velocities $\left[\dot{x}_{k}^{j}, \dot{y}_{k}^{j}\right]$, while the measurement is a noisy version of the position. Hence $M=4$. For 3D calculations, the target state is set as $\tilde{\boldsymbol{m}}_{k}^{j}=\left[x_{k}^{j}, y_{k}^{j}, w_{k}^{j}, \dot{x}_{k}^{j}, \dot{y}_{k}^{j}, \dot{w}_{k}^{j}\right]^{T}$, where $\left[x_{k}^{j}, y_{k}^{j}, w_{k}^{j}\right]$ is the 3D position of the $j$ th target and $\left[\dot{x}_{k}^{j}, \dot{y}_{k}^{j}, \dot{w}_{k}^{j}\right]$ is the target velocity. In Eq. (1) and Eq. (2), $\tau_{k}$ and $\varsigma_{k}$ are system excitation and measurement noise terms, respectively. The clutter term is shown as $\epsilon_{\boldsymbol{k}}$. The transition model and nonlinear measurement model are shown as $\mathbf{F}_{\tilde{m}}$ and $\mathbf{F}_{\boldsymbol{z}}$, respectively.

One of the major issues in multi-target tracking is how to perform an optimal association between multiple measurements and multiple targets. The existing tracking methods can be divided into two kinds: offline and online. Offline tracking uses the past, current and future detections to formulate an optimisation problem. Shen et al. [4] consider the multi-target tracking problem as a submodular maximization problem to find the most related tracklets for trajectory generation. Lan et al. [5] use the interactions between non-associable tracklets to facilitate multi-target tracking and use the efficient quadratic pseudo-Boolean optimization to address the binary labelling problem. Kim et al. [6] use a bilinear long short-term memory (LSTM) to encode jointly the appearance and motion information for target tracking. For online tracking methods, only the past and current detections are used to estimate the target states.

For addressing the multiple measurements problem, several audio-visual multi-speaker tracking methods were proposed in the recent past [7], [8], [9], [10]. Based on the Markov chain Monte Carlo, these papers proposed to use approximate inference of the speaker distribution. The MCMC approach has advantages over the particle filter due to its efficient sampling mechanism. Gilks et al. proposed to use MCMC approach after the resampling step of the particle filter to diversify particles for multiple measurements [10]. However, for multi-speaker tracking, only a few high-weight particles will be retained after resampling due to the weight degeneracy problem. These MCMC based particle filters may miss the speakers, especially for a larger number of measurements. Sequential Markov chain Monte Carlo (SMCMC) [11] is proposed to avoid resampling by sampling directly from the target distribution using rejection sampling. SMCMC based particle filters cannot estimate the number of the speakers. The number of speakers should be given as an initial parameter. However, in the real-world, the number of speakers is unknown and varying.

Recurrent neural networks (RNNs) have been used to encode long-term dependencies across motion, appearance and interaction models to track multiple targets [12]. Zhou et al. [13] proposed a deep continuous conditional random field with unary and asymmetric pairwise terms to model the multitarget appearances and inter-object relations. Chen et al. [14] use deep neural networks to develop a scoring function for candidate selection and adopt person re-identification features for data association.

Discriminative correlation filters (DCFs) have been successfully exploited in multi-target tracking applications due to their high computational efficiency. For example, integrate correlation filters (ICFs) uses a confidence-based relative motion 
network to perform a two-step data association, where CFs are employed as a verifying step to confirm the target estimation [15]. Yang et al. [16] and [17] use multiple single object trackers based on the kernelized correlation filters (KCFs) in parallel for fast tracking. However, the above approaches can be sensitive to false positives, when CFs are performed with unreliable references or labels.

\section{RANDOM FINITE SET}

In classical Bayesian filters, it is often assumed that the target generates one measurement, the number of targets is unchanged, all targets are all detected, and there is no clutter. In practice, one often encounters the situation with the variable number of targets, undetected targets, noisy measurements, and clutters. The theory of random finite sets (RFS) is a natural representation of multi-target states and measurements that enables multi-target filtering by the propagation of the multitarget posterior density [3]. The main idea of the RFS approach is to consider the collection of targets as a set-valued state and the collection of measurements as a set-valued measurement. Therefore, the RFS can handle the problem of estimating multiple targets in the presence of clutters and uncertainty by modelling these set-valued entities as random finite sets [18].

Due to the unknown and time-varying number of targets, the size of the state set is time-varying. The multi-target states and measurements are described as follows. Given the RFS of a set of targets $M_{k-1}$ at time $k-1$, the multi-target state at time $k$ is modelled by [19],

$$
\begin{aligned}
\boldsymbol{M}_{k} & =\boldsymbol{S}_{k}\left(\boldsymbol{M}_{k-1}\right) \cup \boldsymbol{B}_{k}\left(\boldsymbol{M}_{k-1}\right) \cup \boldsymbol{\Gamma}_{k}, \\
\boldsymbol{Z}_{k} & =\boldsymbol{\Theta}_{k}\left(\boldsymbol{M}_{k}\right) \cup \boldsymbol{C}_{k},
\end{aligned}
$$

where ' $U$ ' denotes union, $\boldsymbol{S}_{k}\left(\boldsymbol{M}_{k-1}\right)$ is the RFS of targets that have survived at time $k, \boldsymbol{B}_{k}\left(\boldsymbol{M}_{k-1}\right)$ is the RFS of targets spawned from the previous set of targets $\boldsymbol{M}_{k-1}$ and $\boldsymbol{\Gamma}_{k}$ is the RFS of the new targets that appear at time $k$ [20]. The target RFS encapsulates all aspects of multi-target motion such as the time-varying number of targets, individual target motion, target birth, spawning, and target interactions. The RFSs of the measurements generated by the targets and clutter are denoted by $\boldsymbol{\Theta}_{k}\left(\boldsymbol{M}_{k}\right)$ and $\boldsymbol{C}_{k}$. The measurement RFS encapsulates all sensor characteristics such as measurement noise, sensor field of view and false alarms. The details on how $\boldsymbol{S}_{k}\left(\boldsymbol{M}_{k-1}\right), \boldsymbol{B}_{k}\left(\boldsymbol{M}_{k-1}\right), \boldsymbol{\Gamma}_{k}, \boldsymbol{\Theta}_{k}\left(\boldsymbol{M}_{k}\right)$ and $\boldsymbol{C}_{k}$, are calculated by individual target state, target births, deaths and clutters are available in [21], [22], [23]. However, the RFS approach is computationally intractable since multiple integrals are involved in the recursion of the multi-target posterior.

\section{PHD FILTER}

The PHD filter is a computationally cheaper alternative to the RFS which is the first-order approximation of the RFS and propagates only the first order moments instead of the full multi-target posterior [20], [24], [25], [26], [27], [28], [29], [30]. The PHD filter propagates the intensity function of the multi-target posterior. The integral of the intensity function on any region of the state space gives the expected number of targets, which can be used to estimate the states of individual targets [3]. The PHD filter has two iterative steps: prediction step and update step.

In the prediction step, the PHD of the $j$ th target at time $k$ is defined as [3]

$\Psi_{k \mid k-1}^{j}\left(\boldsymbol{m}_{k}^{j}\right)=\xi_{k}\left(\boldsymbol{m}_{k}^{j}\right)+\int \phi_{k \mid k-1}\left(\boldsymbol{m}_{k}^{j} \mid \boldsymbol{m}_{k-1}^{j}\right) \Psi_{k-1}^{j}\left(\boldsymbol{m}_{k-1}^{j}\right) d \boldsymbol{m}$

where $\xi_{k}\left(\boldsymbol{m}_{k}^{j}\right)$ is the intensity function of the birth RFS $\Gamma_{k}$, and $\phi_{k \mid k-1}\left(\boldsymbol{m}_{k}^{j} \mid \boldsymbol{m}_{k-1}^{j}\right)$ is the analogy of the state transition probability [3]

$\phi_{k \mid k-1}\left(\boldsymbol{m}_{k}^{j} \mid \boldsymbol{m}_{k-1}^{j}\right)=p_{S, k}\left(\boldsymbol{m}_{k-1}^{j}\right) f_{k \mid k-1}\left(\boldsymbol{m}_{k}^{j} \mid \boldsymbol{m}_{k-1}^{j}\right)+\beta_{k \mid k-1}\left(\boldsymbol{m}_{k}^{j}\right.$

where $p_{S, k}\left(\boldsymbol{m}_{k-1}^{j}\right)$ is the survival probability for the targets still existing at time $k$ and $f_{k \mid k-1}\left(\boldsymbol{m}_{k}^{j} \mid \boldsymbol{m}_{k-1}^{j}\right)$ is the singletarget state transition density. The intensity function of spawn is denoted by $\beta_{k \mid k-1}\left(\boldsymbol{m}_{k}^{j} \mid \boldsymbol{m}_{k-1}^{j}\right)$ for the target spawned at time $k$. The update step is defined as [3]

$$
\begin{aligned}
\Psi_{k}^{j}\left(\boldsymbol{m}_{k}^{j}\right)= & {\left[1-p_{D, k}\left(\boldsymbol{m}_{k}^{j}\right)\right] \Psi_{k \mid k-1}^{j}\left(\boldsymbol{m}_{k}^{j}\right) } \\
& +\sum_{\boldsymbol{z}_{k} \in \boldsymbol{Z}_{k}} \frac{p_{D, k}\left(\boldsymbol{m}_{k}^{j}\right) h_{k}\left(\boldsymbol{z}_{k} \mid \boldsymbol{m}_{k}^{j}\right) \Psi_{k \mid k-1}^{j}\left(\boldsymbol{m}_{k}^{j}\right)}{\left.\kappa_{k}\right)+\int p_{D, k}\left(\boldsymbol{m}_{k}^{j}\right) h_{k}\left(\boldsymbol{z}_{k} \mid \boldsymbol{m}_{k}^{j}\right) \Psi_{k \mid k-1}^{j}\left(\boldsymbol{m}_{k}^{j}\right.}
\end{aligned}
$$

where $p_{D, k}\left(\boldsymbol{m}_{k}^{j}\right)$ is detection probability and $h_{k}\left(\boldsymbol{z}_{k} \mid \boldsymbol{m}_{k}^{j}\right)$ is the single-target likelihood defining the probability that $\boldsymbol{z}_{k}$ is generated by a target state $\boldsymbol{m}_{k}^{j}$. The intensity of clutter RFS $\boldsymbol{C}_{k}$ is defined as $\kappa_{k}\left(\boldsymbol{z}_{k}\right)$ which is $\kappa_{k}\left(\boldsymbol{z}_{k}\right)=u \boldsymbol{u}\left(\boldsymbol{z}_{k}\right)$, where $u$ is the average number of clutter points and $u\left(z_{k}\right)$ is the probability distribution of each clutter point.

\section{SMC-PHD FILTER}

To obtain a numerical solution for the integrals in PHD recursion, the SMC method has been proposed which approximates the PHD with a set of random samples (particles) [20]. Suppose that at time step $k-1$, the PHD $\Psi_{k-1}^{j}\left(\boldsymbol{m}_{k-1}^{j}\right)$ is approximated by $\left\{w_{k-1}^{i}, \boldsymbol{m}_{k-1}^{i}\right\}_{i=1}^{N_{k-1}}$ of $N_{k-1}$ particles and their corresponding weights as [20]

$$
\Psi_{k-1}^{j}\left(\boldsymbol{m}_{k-1}^{j}\right) \approx \sum_{i=1}^{N_{k-1}} w_{k-1}^{i} \delta\left(\boldsymbol{m}_{k-1}^{j}-\boldsymbol{m}_{k-1}^{i}\right),
$$

where $\delta$ is the Dirac delta function.

The SMC-PHD filter has two steps: the prediction step and the update step as PHD filter. Prediction of the PHD $\Psi_{k \mid k-1}^{j}\left(\boldsymbol{m}_{k}^{j}\right)$ is obtained with particles $\left\{\boldsymbol{m}_{k-1}^{j}\right\}_{i=1}^{N_{k-1}}$ and their weights $\left\{w_{k-1}^{j}\right\}_{i=1}^{N_{k-1}}$. Here, $N_{k-1}$ particles of $\boldsymbol{M}_{k}$ are first drawn from importance sampling $q_{k}\left(\cdot \mid \boldsymbol{M}_{k-1}, \boldsymbol{Z}_{k}\right)$ to propagate the particles from time step $k-1$. The particle set is obtained by the proposal distribution $q_{k}\left(\boldsymbol{m}_{k \mid k-1}^{i} \mid \boldsymbol{m}_{k-1}^{i}, \boldsymbol{Z}_{k}\right)$,

$$
\boldsymbol{m}_{k \mid k-1}^{i} \propto q_{k}\left(\cdot \mid \boldsymbol{m}_{k-1}^{i}, \boldsymbol{Z}_{k}\right),
$$


Their weights are

$$
\omega_{k \mid k-1}^{i}=\frac{p_{s} \phi_{k}\left(\boldsymbol{m}_{k \mid k-1}^{i} \mid \boldsymbol{m}_{k-1}^{i}\right) \omega_{k-1}^{i}}{q_{k}\left(\boldsymbol{m}_{k \mid k-1}^{i} \mid \boldsymbol{m}_{k-1}^{i}, \boldsymbol{Z}_{k}\right)}, \quad \text { where } i=1, \ldots, N_{k-1},
$$

where $\phi_{k}\left(\boldsymbol{m}_{k \mid k-1}^{i} \mid \boldsymbol{m}_{k-1}^{i}\right)$ is the transition distribution and $p_{s}$ is the survival distribution. Since the proposal distribution is normally assumed to be the same as the transition distribution [31], [32], [33], Eq. (10) can be simplified as:

$$
\omega_{k \mid k-1}^{i}=p_{s} \omega_{k-1}^{i},
$$

$N_{B}$ particles are drawn from the new born importance function $p_{k}\left(\cdot \mid \boldsymbol{Z}_{k}\right)$ to model the state of new targets appearing in the scene [20]

$$
\boldsymbol{m}_{k \mid k-1}^{i} \propto p_{k}\left(\cdot \mid \boldsymbol{Z}_{k}\right)
$$

Their weights are

$\omega_{k \mid k-1}^{i}=\frac{\gamma_{k}\left(\boldsymbol{m}_{k \mid k-1}^{i}\right)}{N_{B} p_{k}\left(\boldsymbol{m}_{k \mid k-1}^{i} \mid \boldsymbol{Z}_{k}\right)}, \quad$ where $\quad i=N_{k-1}+1, \ldots, N_{k-1}+N_{B}$,

where $\gamma_{k}$ is the probability of new born targets, whose integral approximates the average number of targets in the state space. The number of surviving particles at time $k-1$ is denoted by $N_{k-1}$. The $N_{B}$ born particles sampled from the measurements for the born targets at each iteration are added into the particle set and the number of the particles grows over time to make the PHD filter inefficient.

The update step of the PHD recursion is obtained by updating the weight of the predicted particles when the likelihood $h_{k}^{r, i}\left(\boldsymbol{z}_{k}^{r} \mid \boldsymbol{x}_{k}^{i}\right)$ is available. Then $v_{k \mid k-1}\left(\boldsymbol{m}_{k}\right)$ is substituted into Eq. (7) and the predicted weights $\left\{w_{k \mid k-1}^{i}\right\}_{n=1}^{N_{k-1}+N_{B}}$ are updated according to [20]

$$
\omega_{k}^{i}=\left[1-p_{D, k}^{i}+\sum_{r=1}^{R_{k}} \frac{p_{D, k}^{i} h_{k}^{r, i}}{\kappa_{k}+\sum_{i=1}^{N_{k}} p_{D, k}^{i} h_{k}^{r, i} \omega_{k \mid k-1}^{i}}\right] \omega_{k \mid k-1}^{i},
$$

where $p_{D, k}^{i}$ is detection probability and $\kappa_{k}$ is clutter density. These two parameters should be given in the baseline SMCPHD filter. However, in the real world, these two parameters are varying and unknown. In the case that the parameter changes smoothly or is simply time-invariant, the historical data in the past frames can be used to estimate the unknown $p_{D, k}^{i}$ and $\kappa_{k}$. In the case that the parameters are strongly time-variant, only a few of the latest observations can be used to estimate the parameters [34]. Based on the online estimating method, $p_{D, k}^{i}$ and $\kappa_{k}$ can be estimated by the newest measurements [35], [36].

The number of targets is estimated as the sum of the weights,

$$
\tilde{N}_{k}=\sum_{i=1}^{N_{k}} \omega_{k}^{i}
$$

The states and weights of the targets $\left\{\tilde{\boldsymbol{m}}_{k}^{j}, \tilde{\omega}_{k}^{j}\right\}_{j=1}^{\tilde{N}_{k}}$ can be calculated using e.g. K-means clustering method [37] or multi-expected a posterior (MEAP) [32], [38]. Due to the weight degeneracy problem, only a few particles have high weights over several iterations. The low weight particles should be removed and the particles with high weights should be duplicated in order to concentrate the particles on the zones around the estimated targets, which is called the resampling step. After the update step, the resampling is performed when the effective sample size (ESS) [39] is smaller than half of the number of particles. In the resampling step, $N_{k}$ particles are resampled from $\left\{w_{k}^{i} / \Xi_{k}, \boldsymbol{x}_{k}^{i}\right\}_{i=1}^{N_{k-1}+N_{B}}$ where $\Xi_{k}$ is the total mass and $\Xi_{k}=\sum_{i=1}^{N_{k-1}+N_{B}} w_{k}^{i}$. Therefore, the complexity of the SMC-PHD filter increases linearly with the number of targets. After the resampling step, new weights of the set $\left\{w_{k}^{i}, \boldsymbol{x}_{k}^{i}\right\}_{i=1}^{N_{k}}$ are normalized to preserve the total mass.

\section{CONCLUSiON}

Among the obvious signs clarified above, shading signals have been utilized all through this proposal because of their simple usage and low multifaceted nature. Shading data is utilized by taking the histogram of potential focuses at the introduction step as reference pictures and afterward utilizing them in location and following of the objective. In the writing, the RGB or HSV shading histogram model is normally utilized [40]. In our examination, HSV is picked since it is seen to be increasingly powerful to enlightenment variety.

\section{REFERENCES}

[1] C.-Y. Chong, "Tracking and data fusion: A handbook of algorithms," IEEE Control Systems Magazine, vol. 32, no. 5, pp. 114-116, 2012.

[2] S. Blackman and R. Popoli, "Design and analysis of modern tracking systems (artech house radar library)," Artech House, 1999.

[3] R. P. Mahler, Statistical Multisource-Multitarget Information Fusion. Boston, MA, USA: Artech House, 2007.

[4] J. Shen, Z. Liang, J. Liu, H. Sun, L. Shao, and D. Tao, "Multiobject tracking by submodular optimization," IEEE Trans. cybernetics, no. 99, pp. 1-12, 2018.

[5] L. Lan, X. Wang, S. Zhang, D. Tao, W. Gao, and T. S. Huang, "Interacting tracklets for multi-object tracking," IEEE Trans. Image Processing, vol. 27, no. 9, pp. 4585-4597, 2018.

[6] C. Kim, F. Li, and J. M. Rehg, "Multi-object tracking with neural gating using bilinear LSTM," in Proc. the European Con. Computer Vision (ECCV), 2018, pp. 200-215.

[7] N. Checka, K. W. Wilson, M. R. Siracusa, and T. Darrell, "Multiple person and speaker activity tracking with a particle filter," in Proc. IEEE Int. Con. Acoustics, Speech, and Signal Processing, vol. 5, 2004, pp. $881-884$.

[8] D. Gatica-Perez, G. Lathoud, J.-M. Odobez, and I. McCowan, "Audiovisual probabilistic tracking of multiple speakers in meetings," IEEE Trans. Audio, Speech, and Language Processing, vol. 15, no. 2, pp. 601-616, 2007.

[9] T. M. Hospedales and S. Vijayakumar, "Structure inference for bayesian multisensory scene understanding," IEEE Trans. pattern analysis and machine intelligence, vol. 30, no. 12, pp. 2140-2157, 2008.

[10] W. R. Gilks and C. Berzuini, "Following a moving target Monte Carlo inference for dynamic Bayesian models," Journal of the Royal Statistical Society, vol. 63, no. 1, pp. 127-146, 2001.

[11] Z. Khan, T. Balch, and F. Dellaert, "Mcmc-based particle filtering for tracking a variable number of interacting targets," IEEE Trans. Pattern Analysis and Machine Intelligence, vol. 27, no. 11, pp. 1805-1819, 2005.

[12] A. Sadeghian, A. Alahi, and S. Savarese, "Tracking the untrackable: Learning to track multiple cues with long-term dependencies," in Proc. the IEEE Int. Conf. Computer Vision, 2017, pp. 300-311.

[13] H. Zhou, W. Ouyang, J. Cheng, X. Wang, and H. Li, "Deep continuous conditional random fields with asymmetric inter-object constraints for online multi-object tracking," IEEE Trans. Circuits and Systems for Video Technology, 2018.

[14] C. Long, A. Haizhou, Z. Zijie, and S. Chong, "Real-time multiple people tracking with deeply learned candidate selection and person reidentification," vol. 5, p. 8, 2018.

[15] S.-H. Park, K. Lee, and K.-J. Yoon, "Robust online multiple object tracking based on the confidence-based relative motion network and correlation filter," in IEEE Int. Conf. Image Processing (ICIP). IEEE, 2016, pp. 3484-3488. 
[16] Y. Yang and G.-A. Bilodeau, "Multiple object tracking with kernelized correlation filters in urban mixed traffic," in Conf. Computer and Robot Vision (CRV). IEEE, 2017, pp. 209-216.

[17] $\mathrm{H}$. Wu and $\mathrm{W}$. $\mathrm{Li}$, "Robust online multi-object tracking based on KCF trackers and reassignment," in IEEE Global Conf. Signal and Information Processing (GlobalSIP). IEEE, 2016, pp. 124-128.

[18] B.-N. Vo and W.-K. Ma, "A closed-form solution for the probability hypothesis density filter," in Proc. Int. Con. Information Fusion, vol. 2. IEEE, 2005, pp. 856-863.

[19] W.-K. Ma, B.-N. Vo, S. S. Singh, and A. Baddeley, "Tracking an unknown time-varying number of speakers using TDOA measurements: A random finite set approach," IEEE Trans. Signal Processing, vol. 54, no. 9, pp. 3291-3304, 2006.

[20] B.-N. Vo, S. Singh, and A. Doucet, "Sequential Monte Carlo methods for multitarget filtering with random finite sets," IEEE Trans. Aerospace and Electronic Systems, vol. 41, no. 4, pp. 1224-1245, 2005.

[21] I. R. Goodman, R. P. Mahler, and H. T. Nguyen, Mathematics of Data Fusion. Springer Science \& Business Media, 2013, vol. 37.

[22] R. Mahler, An Introduction to Multisource-multitarget Statistics and Applications. Lockheed Martin, 2000.

[23] R. Mahler, D. L. Hall, and J. Llinas, "Random set theory for target tracking and identification." CRC press Boca Raton, 2001, p. 14.

[24] R. P. Mahler, "Multitarget Bayes filtering via first-order multitarget moments," IEEE Trans. Aerospace and Electronic Systems, vol. 39, no. 4, pp. 1152-1178, 2003.

[25] Y. Liu, W. Wang, J. Chambers, V. Kilic, and A. Hilton, "Particle flow smc-phd filter for audio-visual multi-speaker tracking," in International Conference on Latent Variable Analysis and Signal Separation. Springer, 2017, pp. 344-353.

[26] Y. Liu, W. Wang, and Y. Zhao, "Particle flow for sequential monte carlo implementation of probability hypothesis density," in 2017 IEEE International Conference on Acoustics, Speech and Signal Processing (ICASSP). IEEE, 2017, pp. 4371-4375.

[27] Y. Liu, A. Hilton, J. Chambers, Y. Zhao, and W. Wang, "Non-zero diffusion particle flow SMC-PHD filter for audio-visual multi-speaker tracking," Proc. IEEE Int. Conf. Acoustics, Speech and Signal Processing (ICASSP), 2018

[28] Y. Liu, W. Wang, and V. Kilic, "Intensity particle flow smc-phd filter for audio speaker tracking," arXiv preprint arXiv:1812.01570, 2018.

[29] Y. Liu, V. Kılıç, J. Guan, and W. Wang, "Audio-visual particle flow smc-phd filtering for multi-speaker tracking," IEEE Transactions on Multimedia, vol. 22, no. 4, pp. 934-948, 2019.

[30] Y. Liu, Q. Hu, Y. Zou, and W. Wang, "Labelled non-zero particle flow for smc-phd filtering," in ICASSP 2019-2019 IEEE International Conference on Acoustics, Speech and Signal Processing (ICASSP). IEEE, 2019, pp. 5197-5201.

[31] B. Ristic, D. Clark, and B.-N. Vo, "Improved SMC implementation of the PHD filter," in Proc. IEEE Int. Conf. Information Fusion (FUSION), Jul. 2010, pp. 1-8.

[32] T. Li, S. Sun, M. Bolić, and J. M. Corchado, "Algorithm design for parallel implementation of the SMC-PHD filter," Signal Processing, vol. 119, pp. 115-127, 2016.

[33] Y. Liu, W. Wang, J. Chambers, V. Kilic, and A. Hilton, "Particle flow SMC-PHD filter for audio-visual multi-speaker tracking," in Proc. IEEE Intl Conf. Latent Variable Analysis and Signal Separation, Mar. 2017, pp. 344-353.

[34] S. H. Rezatofighi, S. Gould, B. T. Vo, B.-N. Vo, K. Mele, and R. Hartley, "Multi-target tracking with time-varying clutter rate and detection profile: Application to time-lapse cell microscopy sequences," IEEE Tran. medical imaging, vol. 34, no. 6, pp. 1336-1348, 2015.

[35] X. Wang, T. Li, S. Sun, and J. M. Corchado, "A survey of recent advances in particle filters and remaining challenges for multitarget tracking," Sensors, vol. 17, no. 12, p. 2707, 2017.

[36] W. Liu, Y. Chen, H. Cui, and C. Wen, "A nonuniform clutter intensity estimation algorithm for random finite set filters," IEEE Tran. Aerospace and Electronic Systems, vol. 54, no. 6, pp. 2911-2925, 2018.

[37] D. Arthur and S. Vassilvitskii, "K-means++: The advantages of careful seeding," in Proc. Annual ACM-SIAM Symposium on Discrete Algorithms. Society for Industrial and Applied Mathematics, 2007, pp. $1027-1035$.

[38] T. Li, S. Sun, J. M. Corchado, and M. F. Siyau, "A particle dyeing approach for track continuity for the SMC-PHD filter," in Proc. IEEE Int. Conf. Information Fusion (FUSION), Jul. 2014, pp. 7-10.

[39] A. Kong, J. S. Liu, and W. H. Wong, "Sequential imputations and Bayesian missing data problems," Journal of the American Statistical Association, vol. 89, no. 425, pp. 278-288, 1994.
[40] L. Sigal, S. Sclaroff, and V. Athitsos, "Estimation and prediction of evolving color distributions for skin segmentation under varying illumination," in Proc. IEEE Con. Computer Vision and Pattern Recognition, vol. 2, 2000, pp. 152-159. 